\title{
GROWTH CONDITIONS FOR OPERATORS WITH SMALLEST SPECTRUM
}

\author{
H. S. MUSTAFAYEV \\ Department of Mathematics, Faculty of Sciences, Yuzuncu Yil University, \\ Van 65080, Turkey \\ e-mail:hsmustafayev@yahoo.com
}

Dedicated to the memory of professor Mirabbas Gasymov

(Received 23 January 2014; accepted 6 May 2014; first published online

18 December 2014)

\begin{abstract}
Let $A$ be an invertible operator on a complex Banach space $X$. For a given $\alpha \geq 0$, we define the class $\mathcal{D}_{A}^{\alpha}(\mathbb{Z})\left(\right.$ resp. $\mathcal{D}_{A}^{\alpha}\left(\mathbb{Z}_{+}\right)$) of all bounded linear operators $T$ on $X$ for which there exists a constant $C_{T}>0$, such that

$$
\left\|A^{n} T A^{-n}\right\| \leq C_{T}(1+|n|)^{\alpha},
$$

for all $n \in \mathbb{Z}$ (resp. $n \in \mathbb{Z}_{+}$). We present a complete description of the class $\mathcal{D}_{A}^{\alpha}(\mathbb{Z})$ in the case when the spectrum of $A$ is real or is a singleton. If $T \in \mathcal{D}_{A}(\mathbb{Z})\left(=\mathcal{D}_{A}^{0}(\mathbb{Z})\right)$, some estimates for the norm of $A T-T A$ are obtained. Some results for the class $\mathcal{D}_{A}^{\alpha}\left(\mathbb{Z}_{+}\right)$are also given.
\end{abstract}

2010 Mathematics Subject Classification. 47A11, 46Hxx, 30D20.

1. Introduction. Let $X$ be a complex Banach space and let $B(X)$ be the algebra of all bounded linear operators on $X$. As usual, $K(X)$ will denote the ideal of compact operators on $X$. By $\sigma(T), r(T)$, and $R(z, T):=(z I-T)^{-1}(z \notin \sigma(T))$, respectively, we denote the spectrum, the spectral radius, and the resolvent of $T \in B(X)$. Throughout, $[\alpha]$ denotes the integer part of $\alpha \in \mathbb{R}$.

Let $H$ be a separable Hilbert space and let $A$ be an invertible operator on $H$. In [6], Deddens introduced the set

$$
\mathcal{B}_{A}:=\left\{T \in B(H): \sup _{n \geq 0}\left\|A^{n} T A^{-n}\right\|<\infty\right\} .
$$

Notice that $\mathcal{B}_{A}$ is an algebra (not necessarily closed) with identity which contains the commutant $\{A\}^{\prime}$ of $A$. In [6], Deddens showed that if $A$ is a positive operator with the spectral measure $E(\cdot)$, then $\mathcal{B}_{A}$ coincides with the nest algebra associated with the nest $\{E[0, \lambda]: \lambda \geq 0\}$ (recall that every nest algebra arises in this manner). In the same paper, Deddens conjectured that in the infinite dimensional Hilbert case, the equality $\mathcal{B}_{A}=\{A\}^{\prime}$ holds if the spectrum of $A$ is reduced to $\{1\}$. In [16], Roth gave a negative answer to Deddens conjecture. He showed the existence of a quasinilpotent operator $V$ (the Volterra integration operator) for which $\mathcal{B}_{I+V} \neq\{I+V\}^{\prime}$. In [18], Williams proved that if the spectrum of $A \in B(X)$ is reduced to $\{1\}$ and if $T \in B(X)$ satisfies the condition $\sup _{n \in \mathbb{Z}}\left\|A^{n} T A^{-n}\right\|<\infty$, then $A T=T A$. In [7], Drissi and Mbekhta 
improved Williams result by replacing his condition on $A^{-1}$ by the weaker condition $\left\|A^{-n} T A^{n}\right\|=o\left(e^{\varepsilon \sqrt{n}}\right)(n \rightarrow \infty)$, for every $\varepsilon>0$ (see also [8] and [12]).

In this paper, for an invertible operator $A \in B(X)$ and $\alpha \geq 0$, we define the class $\mathcal{D}_{A}^{\alpha}(\mathbb{Z})\left(\right.$ resp. $\mathcal{D}_{A}^{\alpha}\left(\mathbb{Z}_{+}\right)$) of all operators $T \in B(X)$ for which the growth of $\left\|A^{n} T A^{-n}\right\|$ is at most polynomial in $n \in \mathbb{Z}$ (resp. $n \in \mathbb{Z}_{+}$), explicitly, there exists a constant $C_{T}>0$, such that

$$
\left\|A^{n} T A^{-n}\right\| \leq C_{T}(1+|n|)^{\alpha},
$$

for all $n \in \mathbb{Z}$ (resp. $n \in \mathbb{Z}_{+}$). Clearly, both $\mathcal{D}_{A}^{\alpha}(\mathbb{Z})$ and $\mathcal{D}_{A}^{\alpha}\left(\mathbb{Z}_{+}\right)$contains the commutant of $A$. In the case when $\alpha=0$, instead of $\mathcal{D}_{A}^{0}(\mathbb{Z})$ and $\mathcal{D}_{A}^{0}\left(\mathbb{Z}_{+}\right)$we will use the notations $\mathcal{D}_{A}(\mathbb{Z})$ and $\mathcal{D}_{A}\left(\mathbb{Z}_{+}\right)$, respectively. Notice also that $\mathcal{D}_{A}(\mathbb{Z})$ and $\mathcal{D}_{A}\left(\mathbb{Z}_{+}\right)$are algebras (not necessarily closed) with identity.

The main results of the paper can be summarized as follows.

In Section 2, we give a complete characterization (Theorem 2.1) of the class $\mathcal{D}_{A}^{\alpha}(\mathbb{Z})$ in the case when the spectrum of $A$ is real or is a singleton. It is shown (Theorem 2.8) that if $\sigma(A)=\{\lambda\}$ and $K(X) \subset \mathcal{D}_{A}^{\alpha}\left(\mathbb{Z}_{+}\right)$, then $A=\lambda I+N$, where $N$ is nilpotent of degree $\leq[\alpha]+1$. It is shown (Theorem 2.9) also that if $\sigma(A)=\left\{\lambda_{1}, \ldots, \lambda_{n}\right\}$ and $K(X) \subset$ $\mathcal{D}_{A}^{\alpha}\left(\mathbb{Z}_{+}\right)(0 \leq \alpha<1)$, then $\left|\lambda_{1}\right|=\cdots=\left|\lambda_{n}\right|$ and there exist pairwise disjoint (bounded) projections $P_{1}, \ldots, P_{n}$ such that $P_{1}+\cdots+P_{n}=I$ and $A=\lambda_{1} P_{1}+\cdots+\lambda_{n} P_{n}$.

In Section 3, in the case when $T \in D_{A}(\mathbb{Z})$, some estimates for the norm of $A T-$ $T A$ are given (Theorem 3.2).

2. The class $\mathcal{D}_{A}^{\alpha}(\mathbb{Z})$. The first main result of this section is the following.

THEOREM 2.1. Assume that the spectrum of an invertible operator $A \in B(X)$ lies on the real line and $0 \notin \sigma(A)+\sigma(A)$. Then,

$$
\mathcal{D}_{A}^{\alpha}(\mathbb{Z})=\left\{T \in B(X): \sum_{i=0}^{k}(-1)^{i}\left(\begin{array}{c}
k \\
i
\end{array}\right) A^{k-i} T A^{-k+i}=0\right\},
$$

where $k=[\alpha]+1$. In particular, if $0 \leq \alpha<1$, then $\mathcal{D}_{A}^{\alpha}(\mathbb{Z})=\{A\}^{\prime}$.

For the proof, we need some preliminary results.

For arbitrary $T \in B(X)$ and $x \in X$, we define $\rho_{T}(x)$ to be the set of all $\lambda \in \mathbb{C}$ for which there exists a neighbourhood $O_{\lambda}$ of $\lambda$ with $u(z)$ analytic on $O_{\lambda}$ having values in $X$, such that $(z I-T) u(z)=x$ for all $z \in O_{\lambda}$. This set is open and contains the resolvent set $\rho(T)$ of $T$. By definition, the local spectrum of $T$ at $x$, denoted by $\sigma_{T}(x)$, is the complement of $\rho_{T}(x)$, so it is a compact subset of $\sigma(T)$. This object is most tractable if the operator $T$ has the single-valued extension property (in abbreviation SVEP), i.e., for every open set $U$ in $\mathbb{C}$, the only analytic function $f: U \rightarrow X$ for which the equation $(z I-T) f(z)=0$ holds, is the constant function $f \equiv 0$. In that case, for every $x \in X$, there exists a maximal analytic extension of $R(z, T) x$ to $\rho_{T}(x)$. It follows that if $T$ has SVEP, then $\sigma_{T}(x) \neq \emptyset$, whenever $x \neq 0$. It is easy to see that an operator $T \in B(X)$ having spectrum without interior points has the SVEP (see, [5] and [13]).

Let $\omega=\left(\omega_{n}\right)_{n \in \mathbb{Z}}$ be a sequence of real numbers with $\omega_{n} \geq 1$ and $\omega_{n+m} \leq \omega_{n} \omega_{m}$ for all $n, m \in \mathbb{Z}$. We say then that $\omega$ is a weight on $\mathbb{Z}$. The Beurling algebra $\mathcal{A}_{\omega}$, defined by 
the weight $\omega$, is the set of all functions

$$
f(\zeta)=\sum_{n \in \mathbb{Z}} \widehat{f}(n) \zeta^{n}(|\zeta|=1), \text { with }\|f\|_{\omega}=\sum_{n \in \mathbb{Z}}|\widehat{f}(n)| \omega_{n}<\infty .
$$

Notice that $\mathcal{A}_{\omega}$ is a commutative, semisimple Banach algebra with respect to pointwise multiplication. For arbitrary $\varphi \in \mathcal{A}_{\omega}^{*}$, we will write $\varphi=\{\widehat{\varphi}(n)\}_{n \in \mathbb{Z}}$, where $\widehat{\varphi}(n)=\varphi\left(\zeta^{n}\right)$ $(n \in \mathbb{Z})$. We have

$$
\|\varphi\|_{\omega}:=\sup _{n \in \mathbb{Z}} \frac{|\widehat{\varphi}(n)|}{\omega_{n}}<\infty .
$$

The duality being implemented by the formula

$$
\langle\varphi, f\rangle=\sum_{n \in \mathbb{Z}} \widehat{\varphi}(n) \widehat{f}(n) \quad\left(\varphi \in \mathcal{A}_{\omega}^{*}, f \in \mathcal{A}_{\omega}\right)
$$

We say, the weight $\omega$ is regular if

$$
\sum_{n \in \mathbb{Z}} \frac{\log \omega_{n}}{1+n^{2}}<\infty
$$

For example, the weight $\omega_{n}=(1+|n|)^{\alpha}(\alpha \geq 0)$ is regular and it is called polynomial weight. If $\omega$ is a regular weight, then

$$
\lim _{n \rightarrow \infty} \omega_{n}^{\frac{1}{n}}=\lim _{n \rightarrow \infty} \omega_{-n}^{\frac{1}{n}}=1
$$

Consequently, the maximal ideal space of the algebra $\mathcal{A}_{\omega}$ can be identified with $\Gamma:=$ $\{z \in \mathbb{C}:|z|=1\}\left[\mathbf{1 0}\right.$, Chapter III]. Moreover, the algebra $\mathcal{A}_{\omega}$ is regular in the Shilov sense [10, Chapter III and 1, Chapter XII] if and only if the weight $\omega$ is regular. Below, we will assume that $\omega$ is a regular weight.

If $I$ is a closed ideal of $\mathcal{A}_{\omega}$, the hull of $I$ is the set

$$
\operatorname{hull}(I)=\{\xi \in \Gamma: f(\xi)=0, \forall f \in I\}
$$

If $\varphi \in \mathcal{A}_{\omega}^{*}$, then

$$
I_{\varphi}:=\left\{f \in \mathcal{A}_{\omega}: \varphi \cdot f=0\right\}
$$

is a closed ideal of $\mathcal{A}_{\omega}$, where $\varphi \cdot f$ is a functional on $\mathcal{A}_{\omega}$, defined by

$$
\langle\varphi \cdot f, g\rangle=\langle\varphi, f g\rangle, g \in \mathcal{A}_{\omega}
$$

Recall that the support of $\varphi \in \mathcal{A}_{\omega}^{*}$ is defined as follows. For $\xi \in \Gamma$, we let $\xi \notin \operatorname{supp} \varphi$ iff there is a neighbourhood $O_{\xi}$ of $\xi$ such that $\langle\varphi, f\rangle=0$ for all $f \in \mathcal{A}_{\omega}$ with supp $f \subset O_{\xi}$. An equivalent definition for $\operatorname{supp} \varphi$ is that $\xi \in \operatorname{supp} \varphi \operatorname{iff} \varphi \cdot f=0$ implies $f(\xi)=0$. It follows that

$$
\operatorname{supp} \varphi=\operatorname{hull}\left(I_{\varphi}\right), \forall \varphi \in \mathcal{A}_{\omega}^{*}
$$


Given a closed subset $S$ of $\Gamma$, there are two distinguished closed ideals of $\mathcal{A}_{\omega}$ with hull equal to $S$, namely

$$
J_{\omega}(S):=\overline{\left\{f \in \mathcal{A}_{\omega}: \operatorname{supp} f \cap S=\emptyset\right\}}
$$

is the smallest closed ideal whose hull is $S$ and

$$
I_{\omega}(S):=\left\{f \in \mathcal{A}_{\omega}: f(\xi)=0, \forall \xi \in S\right\}
$$

is the largest closed ideal whose hull is $S$. The set $S$ is a set of synthesis for $\mathcal{A}_{\omega}$ if $J_{\omega}(S)=I_{\omega}(S)$. This is equivalent to the existence of a unique closed ideal $I$ of $\mathcal{A}_{\omega}$ whose hull is $S$. It is well known [10, Chapter VI, Section 41] that if $\omega=\left(\omega_{n}\right)_{n \in \mathbb{Z}}$, where $\omega_{n}=(1+|n|)^{\alpha}(0 \leq \alpha<1)$, then each point of $\Gamma$ is a set of synthesis for $\mathcal{A}_{\omega}$.

Let $\varphi \in \mathcal{A}_{\omega}^{*}$ be given. Since $|\widehat{\varphi}(n)| \leq\|\varphi\|_{\omega} \omega_{n}(n \in \mathbb{Z})$, it follows from (1) that

$$
\varlimsup_{n \rightarrow \infty}|\widehat{\varphi}(n)|^{\frac{1}{n}} \leq 1 \text { and } \overline{\lim }_{n \rightarrow \infty}|\widehat{\varphi}(-n)|^{\frac{1}{n}} \leq 1 .
$$

Recall that the Carleman transform of $\varphi$ is defined as the analytic function $\Phi(z)$ on $\mathbb{C} \backslash \Gamma$ given by

$$
\Phi(z)= \begin{cases}\sum_{n=0}^{\infty} \frac{\widehat{\varphi}(n)}{z^{n}}, & |z|>1 \\ -\sum_{n=1}^{\infty} \widehat{\varphi}(-n) z^{n}, & |z|<1 .\end{cases}
$$

We know (see, [2, Theorem 3.3] and [17, Lemma 3]) that $\xi \in \operatorname{supp} \varphi$ if and only if the Carleman transform $\Phi(z)$ of $\varphi$ has no analytic extension to a neighbourhood of $\xi$.

Let $T$ be an invertible operator on a Banach space $X$ and let $\omega=\left(\omega_{n}\right)_{n \in \mathbb{Z}}$ be a weight on $\mathbb{Z}$. We put

$$
E_{T}^{\omega}:=\left\{x \in X: \exists C>0,\left\|T^{n} x\right\| \leq C \omega_{n}, \forall n \in \mathbb{Z}\right\} .
$$

Clearly, $E_{T}^{\omega}$ is a linear (in general, non-closed) subspace of $X$. If $x \in E_{T}^{\omega}$, then for arbitrary $f=\sum_{n \in \mathbb{Z}} \widehat{f}(n) \zeta^{n} \in \mathcal{A}_{\omega}$, we can define $x_{f} \in X$ by

$$
x_{f}=\sum_{n \in \mathbb{Z}} \widehat{f}(n) T^{n} x .
$$

Then, $f \mapsto x_{f}$ is a bounded linear map from $\mathcal{A}_{\omega}$ into $X$;

$$
\left\|x_{f}\right\| \leq C\|f\|_{\omega}, \forall f \in \mathcal{A}_{\omega} .
$$

Further, from the identity

$$
T^{m} x_{f}=\sum_{n \in \mathbb{Z}} \widehat{f}(n) T^{n+m} x,
$$


we can write

$$
\begin{aligned}
\left\|T^{m} x_{f}\right\| & \leq \sum_{n \in \mathbb{Z}}|\widehat{f}(n)|\left\|T^{n+m} x\right\| \\
& \leq C \sum_{n \in \mathbb{Z}}|\widehat{f}(n)| \omega_{n+m} \\
& \leq C\|f\|_{\omega} \omega_{m}, \forall m \in \mathbb{Z} .
\end{aligned}
$$

This shows that $x_{f} \in E_{T}^{\omega}$ for every $f \in \mathcal{A}_{\omega}$. It is easy to check that if $x \in E_{T}^{\omega}$, then

$$
\left(x_{f}\right)_{g}=x_{f g} \text { for all } f, g \in \mathcal{A}_{\omega} \text {. }
$$

It follows that if $x \in E_{T}^{\omega}$, then

$$
I_{x}:=\left\{f \in \mathcal{A}_{\omega}: x_{f}=0\right\}
$$

is a closed ideal of $\mathcal{A}_{\omega}$.

For a given $x \in E_{T}^{\omega}$, consider the function

$$
u(z):= \begin{cases}\sum_{n=0}^{\infty} \frac{T^{n} x}{z^{n+1}}, & |z|>1 \\ -\sum_{n=1}^{\infty} z^{n-1} T^{-n} x, & |z|<1 .\end{cases}
$$

It follows from (1) that $u(z)$ is an analytic function on $\mathbb{C} \backslash \Gamma$ and

$$
(z I-T) u(z)=x(|z| \neq 1) .
$$

It follows that $\sigma_{T}(x) \subset \Gamma$. Now, assume that $T$ has SVEP. We claim that $\sigma_{T}(x)$ consists of all $\xi \in \Gamma$ for which the function $u(z)$ has no analytic extension to a neighbourhood of $\xi$. Assume that $v(z)$ is the analytic extension of $u(z)$ to a neighbourhood $O_{\xi}$ of $\xi \in \Gamma$. It follows from the identity (2) that the function $w(z):=(z I-T) v(z)-x$ vanishes on $O_{\xi}^{+}:=\left\{z \in O_{\xi}:|z|>1\right\}$ and on $O_{\xi}^{-}:=\left\{z \in O_{\xi}:|z|<1\right\}$. By uniqueness theorem, $w(z)=0$ for all $z \in O_{\xi}$. So we have $(z I-T) v(z)=x$ for all $z \in O_{\xi}$. This shows that $\xi \in \rho_{T}(x)$. Now, assume that $\xi \in \rho_{T}(x) \cap \Gamma$. Then, there exists a neighbourhood $O_{\xi}$ of $\xi$ with $v(z)$ analytic on $O_{\xi}$ having values in $X$ such that $(z I-T) v(z)=x$ for all $z \in O_{\xi}$. In view of the identity $(2),(z I-T)(u(z)-v(z))=0$ for all $z \in O_{\xi}^{+}$and $z \in O_{\xi}^{-}$. Since $T$ has SVEP, we have $u(z)=v(z)$ for all $z \in O_{\xi}^{+}$and $z \in O_{\xi}^{-}$. This shows that the function $u(z)$ can be analytically extended to a neighbourhood of $\xi$.

Let $x \in E_{T}^{\omega}$ be given. For arbitrary $\varphi \in X^{*}$, define a functional $\varphi_{x}$ on $\mathcal{A}_{\omega}$, by

$$
\left\langle\varphi_{x}, f\right\rangle=\left\langle\varphi, x_{f}\right\rangle
$$

We have

$$
\left|\left\langle\varphi_{x}, f\right\rangle\right| \leq C\|\varphi\|\|f\|_{\omega}, \forall f \in \mathcal{A}_{\omega}
$$


and $\widehat{\varphi_{x}}(n)=\varphi\left(T^{n} x\right)(n \in \mathbb{Z})$. Consequently, we can write

$$
z\langle\varphi, u(z)\rangle= \begin{cases}\sum_{n=0}^{\infty} \frac{\widehat{\varphi_{x}}(n)}{z^{n}}, & |z|>1 \\ -\sum_{n=1}^{\infty} z^{n} \widehat{\varphi_{x}}(-n), & |z|<1 .\end{cases}
$$

This shows that the function $z \rightarrow z\langle\varphi, u(z)\rangle(|z| \neq 1)$ is the Carleman transform of $\varphi_{x}$. It follows that

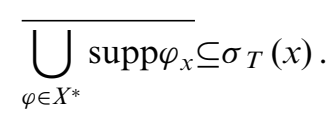

To show the reverse inclusion, assume that $\xi_{0} \in \Gamma$ and

$$
\xi_{0} \notin \overline{\bigcup_{\varphi \in X^{*}} \operatorname{supp} \varphi_{x}} .
$$

Then, there exists $f \in \mathcal{A}_{\omega}$ such that $f\left(\xi_{0}\right) \neq 0$ and $f$ vanishes in a neighbourhood of $\operatorname{supp} \varphi_{x}$, for every $\varphi \in X^{*}$. Consequently, there exists a neighbourhood $O_{\xi_{0}}$ of $\xi$ for which $f(\xi) \neq 0$ for all $\xi \in O_{\xi_{0}}$ and $\varphi_{x} \cdot f=0$. Therefore, $O_{\xi_{0}} \subset \Gamma \backslash \operatorname{supp} \varphi_{x}$. This shows that the function $z \rightarrow\langle\varphi, u(z)\rangle$ can be analytically extended to $O_{\xi_{0}}$ for every $\varphi \in X^{*}$. It follows that $u(z)$ can be analytically extended to $O_{\xi_{0}}$. Consequently, $\xi_{0} \notin \sigma_{T}(x)$. Thus, we obtain

$$
\overline{\bigcup_{\varphi \in X^{*}} \operatorname{supp} \varphi_{x}}=\sigma_{T}(x) .
$$

On the other hand, from the identity

$$
\left\langle\varphi_{x} \cdot f, g\right\rangle=\left\langle\varphi_{x}, f g\right\rangle=\left\langle\varphi, x_{f g}\right\rangle\left(f, g \in \mathcal{A}_{\omega}\right),
$$

we can deduce that

$$
I_{x}=\bigcap_{\varphi \in X^{*}} I_{\varphi_{x}} .
$$

Now, it follows from the general theory of Banach algebras that

$$
\operatorname{hull}\left(I_{x}\right)=\overline{\bigcup_{\varphi \in X^{*}} \operatorname{hull}\left(I_{\varphi_{x}}\right)}=\overline{\bigcup_{\varphi \in X^{*}} \operatorname{supp} \varphi_{x}}=\sigma_{T}(x) .
$$

Hence, we have the following.

PROPOSITION 2.2. Let $\omega=\left(\omega_{n}\right)_{n \in \mathbb{Z}}$ be a regular weight on $\mathbb{Z}$ and let $T$ be an invertible operator on a Banach space $X$ with the SVEP. If $x \in X$ satisfies the condition $\left\|T^{n} x\right\| \leq$ $C \omega_{n}$ for all $n \in \mathbb{Z}$ and for some constant $C>0$, then

$$
\sigma_{T}(x)=\operatorname{hull}\left(I_{x}\right)
$$

As a consequence of Proposition 2.2, we have the following. 
Proposition 2.3. Let $\omega=\left(\omega_{n}\right)_{n \in \mathbb{Z}}$ be a regular weight on $\mathbb{Z}$ and let $T$ be an invertible operator on a Banach $X$ with the SVEP. Assume that $x \in X$ satisfies the condition $\left\|T^{n} x\right\| \leq C \omega_{n}$ for all $n \in \mathbb{Z}$ and for some constant $C>0$. Then, the following assertions hold for $f \in \mathcal{A}_{\omega}$ :

(a) If $x_{f}=0$, then $f$ vanishes on $\sigma_{T}(x)$.

(b) Iff vanishes in a neighbourhood of $\sigma_{T}(x)$, then $x_{f}=0$.

(c) Iff $=1$ in a neighbourhood of $\sigma_{T}(x)$, then $x_{f}=x$.

(d) $\sigma_{T}\left(x_{f}\right) \subset \sigma_{T}(x) \cap$ suppf.

(e) $\sigma_{T}(x) \cap\{\xi \in \Gamma: f(\xi) \neq 0\} \subset \sigma_{T}\left(x_{f}\right)$.

Proof. By Proposition 2.2, we can write

$$
J_{\omega}\left(\sigma_{T}(x)\right) \subset I_{x} \subset I_{\omega}\left(\sigma_{T}(x)\right) .
$$

The assertions (a) and (b) follows from this relation.

(c) Since $f-1$ vanishes in a neighbourhood of $\sigma_{T}(x)$, by (b), $x_{f}=x$.

(d) If $g \in I_{x}$, then $x_{g}=0$. As

$$
\left(x_{f}\right)_{g}=x_{f g}=\left(x_{g}\right)_{f}=0,
$$

we have $g \in I_{x_{f}}$. Hence, $I_{x} \subset I_{x_{f}}$ which implies hull $\left(I_{x_{f}}\right) \subset$ hull $\left(I_{x}\right)$. By Proposition 2.2, $\sigma_{T}\left(x_{f}\right) \subset \sigma_{T}(x)$. On the other hand, if $g \in \mathcal{A}_{\omega}$ vanishes on supp $f$, then $f g=0$. This implies

$$
\left(x_{f}\right)_{g}=x_{f g}=0 \text {. }
$$

Consequently, $I_{\omega}(\operatorname{supp} f) \subset I_{x_{f}}$, so that hull $\left(I_{x_{f}}\right) \subset \operatorname{supp} f$. By Proposition 2.2, $\sigma_{T}\left(x_{f}\right) \subset \operatorname{supp} f$. Thus, we have $\sigma_{T}\left(x_{f}\right) \subset \sigma_{T}(x) \cap \operatorname{supp} f$.

(e) Assume that $\xi \in \sigma_{T}(x), f(\xi) \neq 0$, and $\xi \notin \sigma_{T}\left(x_{f}\right)$. Since the algebra $\mathcal{A}_{\omega}$ is regular, there exists $g \in \mathcal{A}_{\omega}$ such that $g(\xi) \neq 0$ and $g$ vanishes in a neighbourhood of $\sigma_{T}\left(x_{f}\right)$. Consequently, $g$ belongs to the smallest closed ideal of $\mathcal{A}_{\omega}$ whose hull is $\sigma_{T}\left(x_{f}\right)$. By Proposition 2.2, $g \in I_{x_{f}}$ and so

$$
x_{f g}=\left(x_{f}\right)_{g}=0 .
$$

By (a), $f g$ vanishes on $\sigma_{T}(x)$. It follows that $f(\xi)=0$ which contradicts $f(\xi) \neq 0$.

Next, we have the following.

Proposition 2.4. Let $\omega=\left(\omega_{n}\right)_{n \in \mathbb{Z}}$, where $\omega_{n}=(1+|n|)^{\alpha}(\alpha \geq 0)$. Assume that an invertible operator $T$ on a Banach space $X$ and $x \in X$ satisfies the following conditions:

(i) $\left\|T^{n} x\right\| \leq C \omega_{n}$ for all $n \in \mathbb{Z}$ and for some constant $C>0$.

(ii) Thas SVEP.

If $\sigma_{T}(x)=\{\xi\}$, then for every $f \in \mathcal{A}_{\omega}$, we have

$$
x_{f}=f(\xi) x+\frac{f^{\prime}(\xi)}{1 !}(T-\xi I) x+\cdots+\frac{f^{(k)}(\xi)}{k !}(T-\xi I)^{k} x,
$$

where $k=[\alpha]$. In particular, we have $(T-\xi I)^{k+1} x=0$. 
Proof. We know [10, Chapter, Section 41] that if $f \in \mathcal{A}_{\omega}$, then the first $k$ derivatives of $f$ exist and

$$
J_{\omega}(\{\xi\})=\left\{f \in \mathcal{A}_{\omega}: f(\xi)=f^{\prime}(\xi)=\cdots=f^{(k)}(\xi)=0\right\},
$$

where $k=[\alpha]$. Recall that $J_{\omega}(\{\xi\})$ is the smallest closed ideal of $\mathcal{A}_{\omega}$ whose hull is $\{\xi\}$. On the other hand, by Proposition 2.2, hull $\left(I_{x}\right)=\{\xi\}$. Therefore, we have $J_{\omega}(\{\xi\}) \subset I_{x}$. Now, for a given $f \in \mathcal{A}_{\omega}$, consider the function

$$
h(\zeta)=f(\zeta)-f(\xi)-\frac{f^{\prime}(\xi)}{1 !}(\zeta-\xi)-\cdots-\frac{f^{(k)}(\xi)}{k !}(\zeta-\xi)^{k} .
$$

As

$$
h(\xi)=h^{\prime}(\xi)=\cdots=h^{(k)}(\xi)=0,
$$

we have $h \in J_{\omega}(\{\xi\})$, so that $h \in I_{x}$. Thus, we obtain $x_{h}=0$ and so

$$
x_{f}=f(\xi) x+\frac{f^{\prime}(\xi)}{1 !}(T-\xi I) x+\cdots+\frac{f^{(k)}(\xi)}{k !}(T-\xi I)^{k} x .
$$

By taking in the preceding identity $f(\zeta)=(\zeta-\xi)^{k+1}$, we get

$$
(T-\xi I)^{k+1} x=0 .
$$

For a given $A \in B(X)$, by $L_{A}$ and $R_{A}$, respectively, we denote the left and right multiplication operators on $B(X)$;

$$
L_{A} T=A T, R_{A} T=T A, T \in B(X) .
$$

By Lumer-Rosenblum theorem [15, Theorem 10], for arbitrary $A, B \in B(X)$,

$$
\sigma\left(L_{A} R_{B}\right)=\{\lambda \mu: \lambda \in \sigma(A), \mu \in \sigma(B)\} .
$$

Now, we are in a position to prove Theorem 2.1.

Proof of Theorem 2.1. If $T \in \mathcal{D}_{A}^{\alpha}(\mathbb{Z})$, then we can write

$$
\left\|\left(L_{A} R_{A^{-1}}\right)^{n} T\right\| \leq C(1+|n|)^{\alpha}, \quad \forall n \in \mathbb{Z} .
$$

As we have noted above, in that case

$$
\sigma_{L_{A} R_{A^{-1}}}(T) \subset \Gamma
$$

On the other hand, the Lumer-Rosenblum theorem mentioned above and the condition $0 \notin \sigma(A)+\sigma(A)$ implies that

$$
\sigma_{L_{A} R_{A^{-1}}}(T) \subset \sigma\left(L_{A} R_{A^{-1}}\right) \subset \mathbb{R} \backslash\{-1\} .
$$

Consequently, the operator $L_{A} R_{A^{-1}}$ has SVEP and $\sigma_{L_{A} R_{A^{-1}}}(T) \subset\{1\}$. Since $L_{A} R_{A^{-1}}$ has SVEP, $\sigma_{L_{A} R_{A^{-1}}}(T) \neq \emptyset$. So we have $\sigma_{L_{A} R_{A^{-1}}}(T)=\{1\}$. Applying now Proposition 
2.4 to the operator $L_{A} R_{A^{-1}}$ on the space $B(X)$, we get

$$
\left(L_{A} R_{A^{-1}}-I\right)^{k} T=0,
$$

where $k=[\alpha]+1$. This clearly implies

$$
\sum_{i=0}^{k}(-1)^{i}\left(\begin{array}{c}
k \\
i
\end{array}\right) A^{k-i} T A^{-k+i}=0 .
$$

For the reverse inclusion, assume that $T \in B(X)$ satisfies the last equation. Since

$$
\left(L_{A} R_{A^{-1}}-I\right)^{k} T=0(k \geq 1),
$$

we can write

$$
\begin{aligned}
\left\|A^{n} T A^{-n}\right\| & =\left\|\left(L_{A} R_{A^{-1}}\right)^{n} T\right\| \\
& =\left\|T+\left(\begin{array}{c}
n \\
1
\end{array}\right)\left(L_{A} R_{A^{-1}}-I\right) T+\cdots+\left(\begin{array}{c}
n \\
k-1
\end{array}\right)\left(L_{A} R_{A^{-1}}-I\right)^{k-1} T\right\| \\
& =O(1+n)^{k-1} .
\end{aligned}
$$

As $\left(L_{A^{-1}} R_{A}-I\right)^{k} T=0$, similarly we have $\left\|A^{-n} T A^{n}\right\|=O(1+n)^{k-1}$. Hence, $\left\|A^{n} T A^{-n}\right\|=O(1+|n|)^{k-1}(n \in \mathbb{Z})$.

COROLlary 2.5. If the spectrum of an invertible operator $A \in B(X)$ consists of one point, then the conclusion of Theorem 2.1 remains true.

Proof. Assume that $\sigma(A)=\{\lambda\}$, where $\lambda \neq 0$. If $T \in \mathcal{D}_{A}^{\alpha}(\mathbb{Z})$, then $T \in \mathcal{D}_{B}^{\alpha}(\mathbb{Z})$, where $B=\frac{A}{\lambda}$. Since $\sigma(B)=\{1\}$, by Theorem 2.1, we obtain as required.

It follows from Corollary 2.5 that if $\sigma(A)$ consists of one point and $0 \leq \alpha<1$, then $\mathcal{D}_{A}^{\alpha}(\mathbb{Z})=\{A\}^{\prime}$. Note that if $\alpha \geq 1$, then $\mathcal{D}_{A}^{\alpha}(\mathbb{Z}) \neq\{A\}^{\prime}$, in general. To see this, let $A=\left(\begin{array}{ll}1 & 0 \\ 1 & 1\end{array}\right)$ and $T=\left(\begin{array}{ll}0 & 0 \\ 0 & 1\end{array}\right)$ be $2 \times 2$ matrices on 2 -dimensional Hilbert space. We have $\sigma(A)=\{1\}$ and

$$
A^{n} T A^{-n}=[I+n(A-I)] T[I-n(A-I)]=\left(\begin{array}{cc}
0 & 0 \\
-n & 1
\end{array}\right)(n \in \mathbb{N}) .
$$

Similarly, $A^{-n} T A^{n}=\left(\begin{array}{ll}0 & 0 \\ n & 1\end{array}\right)(n \in \mathbb{N})$. So we have

$$
\left\|A^{n} T A^{-n}\right\|=\left(1+|n|^{2}\right)^{\frac{1}{2}} \quad(n \in \mathbb{Z}) .
$$

This shows that $T \in \mathcal{D}_{A}^{1}(\mathbb{Z})$, but $A T \neq T A$.

Recall that an invertible operator $T$ acting on a Banach space is called doubly power bounded if $\sup _{n \in \mathbb{Z}}\left\|T^{n}\right\|<\infty$. Well-known Gelfand's theorem [9] states that if $T$ is doubly power bounded with $\sigma(T)=\{1\}$, then $T=I$.

We include here the following result, which seems to be unnoticed.

Proposition 2.6. Let $\omega=\left(\omega_{n}\right)_{n \in \mathbb{Z}}$, where $\omega(n)=(1+|n|)^{\alpha}(0 \leq \alpha<1)$. Assume that an invertible operator $T$ on a Banach space $X$ and $x \in X$ satisfies the following conditions: 
(i) $\left\|T^{n} x\right\| \leq C \omega(n)$ for all $n \in \mathbb{Z}$ and for some constant $C>0$.

(ii) Thas SVEP.

$$
\begin{array}{r}
\text { If } \sigma_{T}(x)=\left\{\xi_{1}, \ldots, \xi_{k}\right\}\left(\xi_{i} \neq \xi_{j}, i \neq j, i, j=1, \ldots, k\right), \text { then } \\
x \in \operatorname{ker}\left(T-\xi_{1} I\right) \oplus \cdots \oplus \operatorname{ker}\left(T-\xi_{k} I\right) .
\end{array}
$$

Proof. Let $U_{1}, \ldots, U_{k}$ be disjoint neighbourhoods of $\xi_{1}, \ldots, \xi_{k}$, respectively. Let $V_{i}$ be a neighbourhood of $\xi_{i}$ such that $\overline{V_{i}} \subset U_{i}(i=1, \ldots, k)$. Since the algebra $\mathcal{A}_{\omega}$ is regular, there exist functions $f_{1}, \ldots, f_{k}$ in $\mathcal{A}_{\omega}$ such that $f_{i}=1$ on $V_{i}$ and $f_{i}=0$ outside $U_{i}(i=1, \ldots, k)$. We put $f:=f_{1}+\cdots+f_{k}$. Since $f=1$ in a neighbourhood of $\sigma_{T}(x)$, by Proposition 2.3 (c), $x_{f}=x$. So we have $x=x_{1}+\cdots+x_{k}$, where $x_{i}=x_{f_{i}}$ $(i=1, \ldots, k)$. Further, it follows from Proposition 2.3 (d) and (e) that

$$
\left\{\xi_{i}\right\} \subset \sigma_{T}\left(x_{i}\right) \subset \sigma_{T}(x) \cap \operatorname{supp} f_{i}=\left\{\xi_{i}\right\}
$$

Consequently, we have $\sigma_{T}\left(x_{i}\right)=\left\{\xi_{i}\right\}$. Now, it remains to show that if $x \in E_{T}^{\omega}$ with $\sigma_{T}(x)=\{\xi\}$, then $T x=\xi x$. By Proposition 2.2, hull $\left(I_{x}\right)=\{\xi\}$. Since $\{\xi\}$ is a set of synthesis for $\mathcal{A}_{\omega}$, we have $I_{x}=I_{\omega}(\{\xi\})$, so that

$$
\left\{f \in \mathcal{A}_{\omega}: x_{f}=0\right\}=\left\{f \in \mathcal{A}_{\omega}: f(\xi)=0\right\} .
$$

If we put in this identity $f(\zeta)=\zeta-\xi$, then we have $T x=\xi x$.

Let $\omega=\left(\omega_{n}\right)_{n \in \mathbb{Z}}$ be a regular weight. Assume that an invertible operator $T$ on a Banach space satisfies the condition $\left\|T^{n}\right\| \leq C \omega(n)$ for all $n \in \mathbb{Z}$ and for some constant $C>0$. As we have noted above, in that case $\sigma(T) \subset \Gamma$ and therefore $T$ has SVEP.

The following result is an immediate consequence of the preceding proposition.

Corollary 2.7. Assume that $0 \leq \alpha<1$ and $T \in B(X)$ satisfies the condition $\left\|T^{n}\right\| \leq C(1+|n|)^{\alpha}$ for all $n \in \mathbb{Z}$ and for some constant $C>0$. If

$$
\sigma(T)=\left\{\xi_{1}, \ldots, \xi_{k}\right\}\left(\xi_{i} \neq \xi_{j}, i \neq j, i, j=1, \ldots, k\right),
$$

then there exist pairwise disjoint (bounded) projections $P_{1}, \ldots, P_{k}$ such that $P_{1}+\cdots+$ $P_{k}=I$ and

$$
T=\xi_{1} P_{1}+\cdots+\xi_{k} P_{k}
$$

(in fact, $P_{i}=\frac{1}{2 \pi i} \int_{\Gamma_{i}} R(z, T) d z$, where $\Gamma_{i}$ is an appropriate contour around $\left\{\xi_{i}\right\}$ ).

Another application of Proposition 2.4 is the following.

THEOREM 2.8. Assume that the spectrum of $A \in B(X)$ consists of one point $\lambda \neq 0$. If $K(X) \subset \mathcal{D}_{A}^{\alpha}\left(\mathbb{Z}_{+}\right)$, then the operator $A$ has the form $A=\lambda I+N$, where $N$ is nilpotent of degree $\leq[\alpha]+1$.

Proof. We have

$$
\left\|A^{n} T A^{-n}\right\|=\left\|\left(L_{A} R_{A^{-1}}\right)^{n} T\right\| \leq C_{T}(1+n)^{\alpha},
$$


for all $T \in K(X)$ and $n \in \mathbb{N}$. Applying uniform boundedness principle to the sequence of operators

$$
B_{n}:=\frac{1}{(1+n)^{\alpha}}\left(L_{A} R_{A^{-1}}\right)^{n},
$$

we obtain that there exists a constant $C>0$ such that

$$
\left\|A^{n} T A^{-n}\right\| \leq C(1+n)^{\alpha}\|T\|,
$$

for all $T \in K(X)$ and $n \in \mathbb{N}$. For a given $x \in X$ and $\varphi \in X^{*}$, let $x \otimes \varphi$ be the one dimensional operator on $X$ defined by

$$
x \otimes \varphi: y \mapsto \varphi(y) x(y \in X) .
$$

As $x \otimes \varphi \in \mathcal{D}_{A}^{\alpha}\left(\mathbb{Z}_{+}\right)$, we have

$$
\left\|A^{n} x\right\|\left\|A^{*-n} \varphi\right\| \leq C(1+n)^{\alpha}\|x\|\|\varphi\|,
$$

for all $x \in X$ and $\varphi \in X^{*}$. This implies

$$
\left\|A^{n}\right\|\left\|A^{-n}\right\| \leq C(1+n)^{\alpha}, \quad \forall n \in \mathbb{N} .
$$

Further if $B:=\frac{1}{\lambda} A$, then

$$
\left\|B^{n}\right\|\left\|B^{-n}\right\|=\left\|A^{n}\right\|\left\|A^{-n}\right\| \leq C(1+n)^{\alpha} .
$$

On the other hand, as $\sigma(B)=\sigma\left(B^{-1}\right)=\{1\}$, we have $\left\|B^{n}\right\| \geq 1$ and $\left\|B^{-n}\right\| \geq 1$. Consequently,

$$
\left\|B^{n}\right\| \leq\left\|B^{n}\right\|\left\|B^{-n}\right\| \leq C(1+n)^{\alpha}, \quad \forall n \in \mathbb{N} .
$$

Similarly, we have

$$
\left\|B^{-n}\right\| \leq C(1+n)^{\alpha}, \quad \forall n \in \mathbb{N} .
$$

Thus, we obtain

$$
\left\|B^{n}\right\| \leq C(1+|n|)^{\alpha}, \quad \forall n \in \mathbb{Z} .
$$

By Proposition 2.4, $(B-I)^{k}=0$, where $k=[\alpha]+1$. It follows that $(A-\lambda I)^{k}=0$. If we put $N:=A-\lambda I$, then $A=\lambda I+N$, where $N$ is nilpotent of degree $\leq k$.

The following result is an application of Proposition 2.6.

TheOREM 2.9. Let $A \in B(X)$ be such that $\sigma(A)=\left\{\lambda_{1}, \ldots, \lambda_{k}\right\}$, where $0 \neq \lambda_{i} \neq \lambda_{j}$ $(i \neq j)(i, j=1, \ldots, k)$. If $K(X) \subset \mathcal{D}_{A}^{\alpha}\left(\mathbb{Z}_{+}\right)(0 \leq \alpha<1)$, then $\left|\lambda_{1}\right|=\cdots=\left|\lambda_{k}\right|$ and there exist pairwise disjoint (bounded) projections $P_{1}, \ldots, P_{k}$ such that $P_{1}+\cdots+P_{k}=I$ and

$$
A=\lambda_{1} P_{1}+\cdots+\lambda_{k} P_{k}
$$

Proof. As in the proof of Theorem 2.8, we have

$$
\left\|A^{n}\right\|\left\|A^{-n}\right\| \leq C(1+n)^{\alpha}, \forall n \in \mathbb{N} .
$$


It follows that $r(A) r\left(A^{-1}\right) \leq 1$. Since $r(A) r\left(A^{-1}\right) \geq 1$, we obtain

$$
r(A) r\left(A^{-1}\right)=1 \text {. }
$$

Consequently, $\left|\lambda_{1}\right|=\cdots=\left|\lambda_{k}\right|=a$ for some $a>0$. Further if $B:=\frac{1}{a} A$, then

$$
\left\|B^{n}\right\|\left\|B^{-n}\right\|=\left\|A^{n}\right\|\left\|A^{-n}\right\| \leq C(1+n)^{\alpha}, \quad \forall n \in \mathbb{N} .
$$

On the other hand, as

$$
\sigma(B)=\left\{\frac{\lambda_{1}}{a}, \ldots, \frac{\lambda_{k}}{a}\right\} \quad \text { and } \quad \sigma\left(B^{-1}\right)=\left\{\frac{a}{\lambda_{1}}, \ldots, \frac{a}{\lambda_{k}}\right\},
$$

we have $\left\|B^{n}\right\| \geq 1$ and $\left\|B^{-n}\right\| \geq 1$. This implies

$$
\left\|B^{n}\right\| \leq\left\|B^{n}\right\|\left\|B^{-n}\right\| \leq C(1+n)^{\alpha}, \quad \forall n \in \mathbb{N} .
$$

Similarly, we have

$$
\left\|B^{-n}\right\| \leq C(1+n)^{\alpha}, \quad \forall n \in \mathbb{N}
$$

Thus, we obtain

$$
\left\|B^{n}\right\| \leq C(1+|n|)^{\alpha}, \quad \forall n \in \mathbb{Z} .
$$

By Corollary 2.7, there exist pairwise disjoint projections $P_{1}, \ldots, P_{k}$ such that $P_{1}+$ $\cdots+P_{k}=I$ and

$$
B=\frac{\lambda_{1}}{a} P_{1}+\cdots+\frac{\lambda_{k}}{a} P_{k}
$$

So we have $A=\lambda_{1} P_{1}+\cdots+\lambda_{k} P_{k}$.

3. The norm of $A T-T A$. In this section, we give some estimates for the norm of $A T-T A$ in the case when $T \in \mathcal{D}_{A}(\mathbb{R})$.

Recall that an entire function $f$ is said to be of order $\rho$ if

$$
\rho=\varlimsup_{r \rightarrow \infty} \frac{\log \log M_{f}(r)}{\log r},
$$

where

$$
M_{f}(r)=\sup \{|f(z)|:|z| \leq r\} \quad(r>0) .
$$

An entire function $f$ of finite order $\rho$ is said to be of type $\sigma$ if

$$
\sigma=\varlimsup_{r \rightarrow \infty} \frac{\log M_{f}(r)}{r^{\rho}} .
$$

If the entire function $f$ is of order at most one and type less than or equal to $\sigma$, we say $f$ is of exponential type $\sigma[3$, p. 8].

For a given $\sigma>0$, we denote by $B_{\sigma}$ the set of all bounded on the real line entire functions $f$ of exponential type $\leq \sigma$. Recall [11] that $B_{\sigma}$ is a Banach space under the 
norm given by

$$
\|f\|_{\sigma}:=\sup _{z \in \mathbb{C}}\left[e^{-\sigma|\operatorname{Im} z|}|f(z)|\right] .
$$

It follows from the Phragmen-Lindelöf theorem that

$$
\|f\|_{\sigma}=\sup _{t \in \mathbb{R}}|f(t)|, \quad \forall f \in B_{\sigma} .
$$

The following inequality of Bernstein type is well known [11]: If $f \in B_{\sigma}$, where $0 \leq$ $\sigma h \leq \frac{\pi}{2}$, then

$$
\sup _{t \in \mathbb{R}}|f(t+h)-f(t-h)| \leq 2 \sin \sigma h\|f\|_{\sigma} .
$$

It follows that for every $f \in B_{\sigma}$,

$$
\begin{gathered}
|f(1)-f(0)| \leq 2 \sin \frac{\sigma}{2}\|f\|_{\sigma} \quad(\sigma \leq \pi), \\
|f(1)-f(-1)| \leq 2 \sin \sigma\|f\|_{\sigma} \quad\left(\sigma \leq \frac{\pi}{2}\right) .
\end{gathered}
$$

On the other hand, by Cartwright theorem (see, [3, Chapter 10] and [11]), the inequality

$$
\|f\|_{\sigma} \leq \frac{1}{\cos \frac{\sigma}{2}} \sup _{n \in \mathbb{Z}}|f(n)|
$$

holds for every $f \in B_{\sigma}(\sigma<\pi)$. Hence, we have

$$
\begin{gathered}
|f(1)-f(0)| \leq 2 \tan \frac{\sigma}{2}\left(\sup _{n \in \mathbb{Z}}|f(n)|\right), \forall f \in B_{\sigma}(\sigma<\pi), \\
|f(1)-f(-1)| \leq 4 \sin \frac{\sigma}{2}\left(\sup _{n \in \mathbb{Z}}|f(n)|\right), \forall f \in B_{\sigma} \quad\left(\sigma \leq \frac{\pi}{2}\right) .
\end{gathered}
$$

We will need the following.

Lemma 3.1. Assume that $T \in B(X)$ and $x \in X$ satisfies the following conditions,

(i) $\sigma(T) \subset \mathbb{C} \backslash \mathbb{R}_{-}$,

(ii) $\sup _{n \in \mathbb{Z}}\left\|T^{n} x\right\| \leq C$ for some $C>0$.

If $\tau_{T}:=\sup \{|\log z|: z \in \sigma(T)\}$, then the following assertions hold:

(a) If $\tau_{T}<\pi$, then

$$
\|T x-x\| \leq 2 C \tan \frac{\tau_{T}}{2} .
$$

(b) If $\tau_{T} \leq \frac{\pi}{2}$, then

$$
\left\|T x-T^{-1} x\right\| \leq 4 C \sin \frac{\tau_{T}}{2} .
$$


Proof. By condition (i), we can write $T=e^{S}$, where $S=\log T$ [4, Chapter I, Section 7]. For arbitrary functional $\varphi \in X^{*}$ with norm one, consider the entire function

$$
f(z):=\left\langle\varphi, e^{z S} x\right\rangle
$$

From the inequality,

$$
|f(z)| \leq e^{|z|\|S\|}\|x\|,
$$

we deduce that $f$ is an entire function of order

$$
\rho=\varlimsup_{r \rightarrow \infty} \frac{\log \log M_{f}(r)}{\log r} \leq \lim _{r \rightarrow \infty} \frac{\log (r\|S\|+\log \|x\|)}{\log r}=1 .
$$

Notice also that the $n$th derivative of $f$ at zero is $\varphi\left(S^{n} x\right)$. By Levin's Theorem $[14$, p. 84], the type of $f$ is less than or equal to

$$
\begin{aligned}
\varlimsup_{n \rightarrow \infty}\left|\varphi\left(S^{n} x\right)\right|^{\frac{1}{n}} & \leq \lim _{k \rightarrow \infty}\left\|S^{n}\right\|^{\frac{1}{n}}=r(S) \\
& =\sup \{|\log z|: z \in \sigma(T)\}=\tau_{T} .
\end{aligned}
$$

Consequently, $f$ is an entire function of exponential type $\tau_{T}$. Further, since

$$
\sup _{n \in \mathbb{Z}}\left\|e^{n S} x\right\| \leq C,
$$

from the identity $t=n+r$, where $n \in \mathbb{Z},|r|<1$, and $|n| \leq|t|$, we can write

$$
\sup _{t \in \mathbb{R}}\left\|e^{t S} x\right\| \leq C e^{\|S\|}
$$

Hence, $f$ is bounded on $\mathbb{R}$. Thus, we obtain that $f \in B_{\tau_{T}}$. Now, taking into account that

$$
\sup _{n \in \mathbb{Z}}|f(n)| \leq C,
$$

in the case when $\tau_{T}<\pi$, from the inequality (3), we can write

$$
|f(1)-f(0)| \leq 2 C \tan \frac{\tau_{T}}{2} .
$$

It follows that

$$
\left\|e^{S} x-x\right\| \leq 2 C \tan \frac{\tau_{T}}{2}
$$

which means that

$$
\|T x-x\| \leq 2 C \tan \frac{\tau_{T}}{2} .
$$

Similarly, from the inequality (4), we can deduce that if $\tau_{T} \leq \frac{\pi}{2}$, then

$$
\left\|T x-T^{-1} x\right\| \leq 4 C \sin \frac{\tau_{T}}{2} .
$$


The following theorem gives us another generalization of Williams result [18].

THEOREM 3.2. Let $A$ be an invertible operator on a Banach space $X$ and let $T \in$ $B(X)$. Assume that the following conditions are satisfied:

(i) $\left\{\lambda \mu^{-1}: \lambda, \mu \in \sigma(A)\right\} \subset \mathbb{C} \backslash \mathbb{R}_{-}$,

(ii) $\sup _{n \in \mathbb{Z}}\left\|A^{n} T A^{-n}\right\| \leq C_{T}$ for some $C_{T}>0$.

If $\tau_{A}:=\sup \left\{\left|\log \left(\lambda \mu^{-1}\right)\right|: \lambda, \mu \in \sigma(A)\right\}$, then the following assertions hold:

(a) If $\tau_{A}<\pi$, then

$$
\|A T-T A\| \leq 2 C_{T}\|A\| \tan \frac{\tau_{A}}{2} .
$$

(b) If $\tau_{A} \leq \frac{\pi}{2}$, then

$$
\left\|A^{2} T-T A^{2}\right\| \leq 2 C_{T}\|A\|^{2} \sin \frac{\tau_{A}}{2} .
$$

Proof. We have

$$
\sup _{n \in \mathbb{Z}}\left\|\left(L_{A} R_{A^{-1}}\right)^{n} T\right\| \leq C_{T}
$$

By Lumer-Rosenblum theorem mentioned above, we also have

$$
\sigma\left(L_{A} R_{A^{-1}}\right)=\left\{\lambda \mu^{-1}: \lambda, \mu \in \sigma(A)\right\} \subset \mathbb{C} \backslash \mathbb{R}_{-} .
$$

Applying now Lemma 3.1 to the operator $L_{A} R_{A^{-1}}$ on the space $B(X)$, we can write

$$
\begin{aligned}
\|A T-T A\| & =\left\|\left(A T A^{-1}-T\right) A\right\| \\
& \leq\|A\|\left\|\left(L_{A} R_{A^{-1}}\right) T-T\right\| \\
& \leq 2 C_{T}\|A\| \tan \frac{\tau_{A}}{2} .
\end{aligned}
$$

Similarly, we have

$$
\begin{aligned}
\left\|A^{2} T-T A^{2}\right\| & =\left\|A\left(A T A^{-1}-A^{-1} T A\right) A\right\| \\
& \leq\|A\|^{2}\left\|\left(L_{A} R_{A^{-1}}\right) T-\left(L_{A^{-1}} R_{A}\right) T\right\| \\
& \leq 4 C_{T}\|A\|^{2} \sin \frac{\tau_{A}}{2} .
\end{aligned}
$$

\section{REFERENCES}

1. B. Beauzamy, Introduction to operator theory and invariant subspaces (North-Holland, Amsterdam, 1988).

2. J. Benedetto, Harmonic analysis on totally disconnected sets, Lecture Notes in Mathematics, vol. 202, (Springer, Berlin-Heidelberg-New York, 1971).

3. R. P. Boas, Entire functions (Academic Press, New York, 1954). 1973).

4. F. F. Bonsall and J. Duncan, Complete normed algebras, vol. 80, (Springer-Verlag, Berlin,

5. I. Colojoară and C. Foiaş, Theory of generalized spectral operators (Gordon and Breach, New York, 1968). 
6. J. A. Deddens, Another description of nest algebras in Hilbert spaces operators, Lect. Notes Math. 693 (1978), 77-86.

7. D. Drissi and M. Mbekhta, Operators with bounded conjugation orbits, Proc. Am. Math. Soc. 128 (2000), 2687-2691.

8. D. Drissi and M. Mbekhta, Elements with generalized bounded conjugation orbits, Proc. Am. Math. Soc. 129 (2001), 2011-2016.

9. I. M. Gelfand, Zur theorie der charactere der abelschen topologischen gruppen, Rec. Math. N. S. (Mat. Sb), 51 (1941), 49-50.

10. I. Gelfand, D. Raikov and G. Shilov, Commutative normed rings (Chelsea Publ. Company, New York, 1964).

11. E. A. Gorin, Bernstein's inequality from the point of view of operator theory, Selecta Math. Sov. 7 (1988), 191-209 (transl. from Vestnik Kharkov Univ. 45 (1980), 77-105).

12. M. T. Karaev and H. S. Mustafayev, On some properties of Deddens algebras, Rocky Mt. J. Math. 33 (2003), 915-926.

13. K. B. Laursen and M. Neuman, An introduction to the local spectral theory (Oxford, Clarendon Press, 2000).

14. B. Ya. Levin, Distributions of zeros of entire functions, Amer. Math. Soc. Providence (1964).

15. G. Lumer and M. Rosenblum, Linear operators equations, Proc. Am. Math. Soc. 10 (1959), 32-41.

16. P. G. Roth, Bounded orbits of conjugation, analytic theory, Indiana Univ. Math. J. 32 (1983), 491-509.

17. J. Wermer, The existence of invariant subspaces, Duke Math. J. 19 (1952), 615-622.

18. J. P. Williams, On a boundedness condition for operators with a singleton spectrum, Proc. Am. Math. Soc. 78 (1980), 30-32. 